\title{
Message from the Editor-in-Chief: Theranostic Imaging in 2018
}

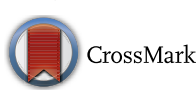

\author{
Dong Soo Lee ${ }^{1}$
}

Published online: 6 April 2018

(C) Korean Society of Nuclear Medicine 2018

In 2018 we are witnessing the progress of theranostic imaging of image-guided therapy in nuclear medicine. We are also now wondering whether the use of nuclear medicine will increase globally or decrease. So far, since the millennium, hybrid imaging and thorough exploration of the use of nuclear medicine has widened its clinical applications (Fig. 1).

Unlike thyroid radionuclide therapy, which does not need individual dosimetry, newly adopted theranostics are sure to become more widely used in the next decade while personalized therapy is mandatory with theranostic imaging. Further progress needs to be made in the treatment of many cancers besides castration-resistant prostate cancer and neuroendocrine tumors (NET) with optimization of therapy/diagnostic imaging. However, this is just the beginning, and advances in cardio/cerebrovascular theranostics should follow the success of NET theranostics as a promising trial in collaboration with cardiologists and neurologists. Atherosclerosis will be tackled with new methods of treatment using kinase inhibitors/monoclonal antibodies and peptides/nanomaterials such as ceria, and this will be accompanied by theranostic imaging. ${ }^{18} \mathrm{~F}$-FDG or ${ }^{18} \mathrm{~F}$-fluoride or ${ }^{99 \mathrm{~m}} \mathrm{Tc}$-annexin images have been introduced, but have not successfully entered popular clinical use. Then, considering brain theranostics, can it (that is, brain theranostics) be formulated for use in the management not only of brain tumors but also of neurodegenerative diseases? What kind of therapeutic modality will be a disruptive innovation in this currently imaginary field of associating 'brain therapy and imaging'?

Hybrid imaging should find its own way of positioning itself with the rapidly developing clinical biomarker studies such as circulating DNA (or RNA and nucleic acids in the exosomes) and circulating tumor cells. There are hopes and worries regarding the future of hybrid imaging including PET/

Dong Soo Lee

dsl@snu.ac.kr

1 Seoul National University, Seoul, South Korea
CT, PET/MRI and SPECT/CT, as they either will prosper together in this "brave new world of popular use of liquid biopsy especially point-of-care applications on site by the practicing clinicians" or will be supplanted by much less time-consuming and easy and cheap liquid biopsy biomarkers and become obsolete. As usual in the history of nuclear medicine, at the height of the use of imaging for everyday routine, we need to make our best effort to predict the near future of our discipline and find the breakthroughs.

In our journal of Nuclear Medicine and Molecular Imaging, as I announced last year, we have tried to answer these questions with the help of our Associate Editors. They joined this effort to depict the current status of the use of nuclear medicine and we are expecting a series of review articles which have been organized by Joon Young Choi for the April and June issues and by Byeong-Cheol Ahn for the August and October issues. Choi invited the reviews and discussion of prospects with the topic "radiomics and deep learning in imaging" and has coordinated the publication of summaries of current trends. This description of radiomics will span from the current effort to the expected future of using deep learning and even how new assisting tools (e.g. artificial intelligence) are going to be harnessed. Ahn has invited summaries and discussion of prospects of the therapy in nuclear medicine for the year of 2018 and onwards. Various aspects of attacking intractable malignancies will be sketched in these reviews, and consensus reports may also be included, for example the use of ${ }^{131}$ I-MIBG.

The global success of clinical nuclear medicine will be achieved by finding the right future direction from the above exploration and innovations while the advantages of our discipline are maximized. Its use will be expanded and propagated by encouraging leaders in the field in developing countries to join the effort to use current state-of-the-art nuclear medicine and to develop customized clinical applications. The latter will require a visionary and nonprofit approach and also heroic efforts by the current global leaders in the field of nuclear medicine. We consider that the current leadership of global organizations such as the Society of Nuclear Medicine (of the 


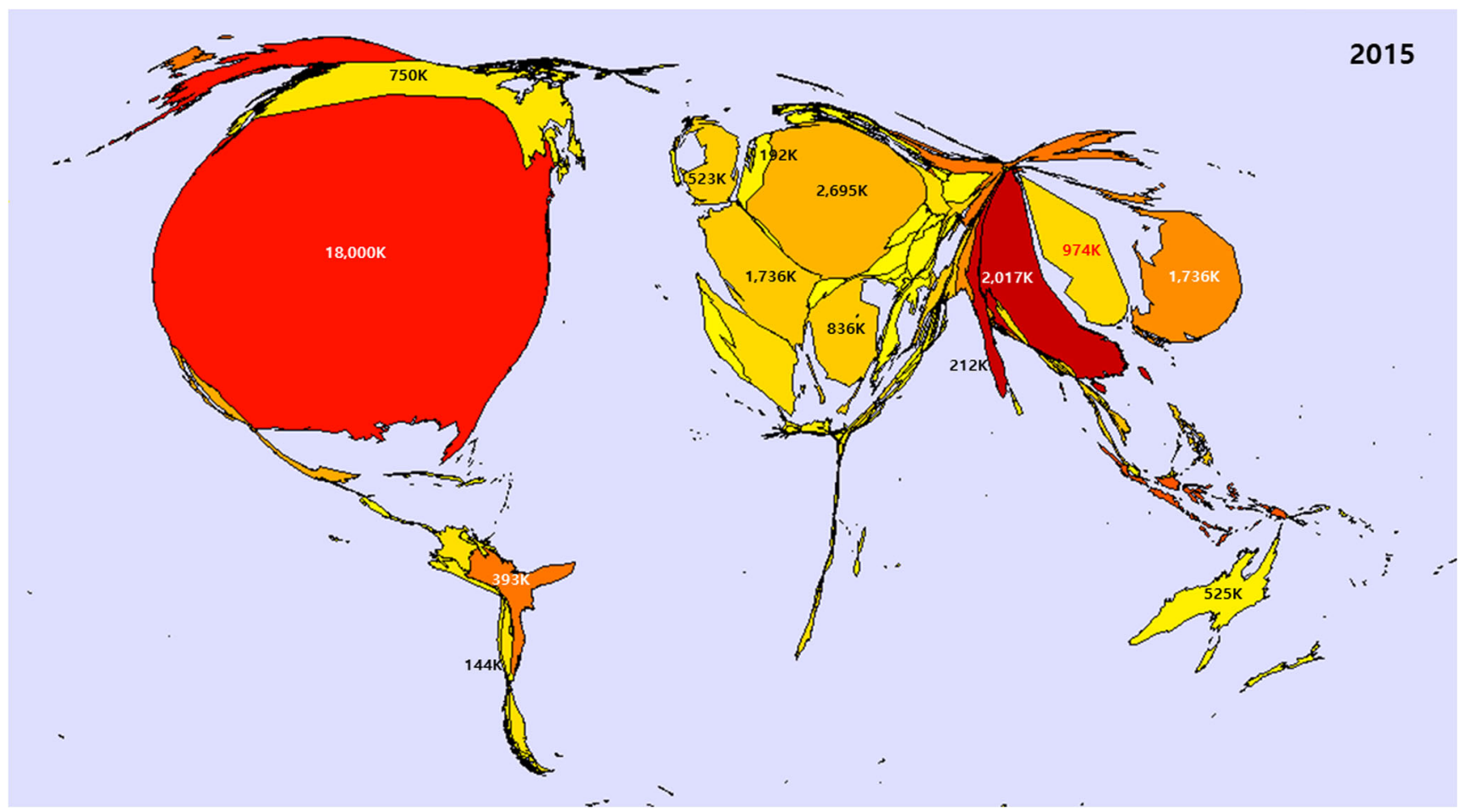

Fig. 1 The number of clinical nuclear imaging and therapy procedures worldwide in the year 2015. Each country's area is transformed to represent the annual number of clinical nuclear medicine and therapy procedures

USA), the European Association of Nuclear Medicine, the International Atomic Energy Agency and the World Federation of Nuclear Medicine and Biology should devise and play their own roles. The endeavor to share any success of their own is better than nothing. Our journal
Nuclear Medicine and Molecular Imaging supported by the intense desire of members of the Korean Society of Nuclear Medicine will tread first to try to share not for prosperity but for the survival of our discipline to the benefit of patients and the wider population. 